\title{
Enfermagem COVID-19: desenvolvimento de um aplicativo Android para suporte ao
} raciocínio clínico de enfermeiras

\author{
COVID-19 Nursing: development of an Android application to support nurses' clinical reasoning \\ Enfermería COVID-19: desarrollo de una aplicación Android para apoyar el razonamiento clínico
}

de las enfermeras

Recebido: 07/10/2021 | Revisado: 12/10/2021 | Aceito: 13/10/2021 | Publicado: 16/10/2021

\author{
Silvio Cesar da Conceição \\ ORCID: https://orcid.org/0000-0003-4745-3333 \\ Universidade Federal do Estado do Rio de Janeiro, Brasil \\ E-mail: silvioenfermeiro73@gmail.com \\ Ádane Viana \\ ORCID: https://orcid.org/0000-0001-9783-2716 \\ Secretaria Municipal de Saúde do Rio de Janeiro, Brasil \\ E-mail: adaneviana@gmail.com \\ Gabriel Conde y Martin Cebriano \\ ORCID: https://orcid.org/0000-0002-0125-7874 \\ Instituto Brasileiro de Medicina de Reabilitação, Brasil \\ E-mail: cebrianogabriel@gmail.com \\ Teresa Tonini \\ ORCID: https://orcid.org/0000-0002-5253-2485 \\ Universidade Federal do Estado do Rio de Janeiro, Brasil \\ E-mail: ttonini@ terra.com.br
}

\begin{abstract}
Resumo
Considerando o caráter de emergência de saúde pública da pandemia; a urgência para se ajustar e repensar os cuidados de enfermagem, com vistas a se debelar ou minimizar os agravos clínicos das pessoas acometidas por COVID-19; e a importância da tomada de decisão clínica dos enfermeiros de forma rápida, eficiente e segura, o objetivo foi desenvolver um aplicativo em SO Android capaz de fornecer suporte ao raciocínio clínico de enfermeiras, baseado nas etapas do processo de enfermagem para pacientes acometidos pela COVID-19. Estudo metodológico, utilizando o PMBOK para o desenvolvimento de aplicativo, seguindo as etapas organizadas em cinco grupos de processos: 1. Iniciação; 2. Planejamento; 3. Execução; 4. Monitoramento; e 5. Encerramento. O desenvolvimento do projeto do aplicativo ocorreu entre os meses de maio e junho de 2020. Os resultados e discussões mostram, em cada etapa, a viabilidade do desenvolvimento do aplicativo, recursos necessários e definição do sistema operacional de suporte; o escopo do aplicativo foi definido de maneira a possibilitar o encadeamento de ideias sobre a assistência de enfermagem; e a execução das atividades necessárias ao alcance dos objetivos do projeto; as estratégias de monitoramento de desempenho. Conclui-se que se cumpriu o objetivo e que o conhecimento dos autores sobre programação de sistema de informação e saber-fazer de Enfermagem foi fundamental para a criação e projeção do aplicativo Enfermagem COVID-19, em tempo veloz como requeria a emergência da pandemia e de acordo com as necessidades das enfermeiras.
\end{abstract}

Palavras-chave: Enfermagem; Processo de enfermagem; Design de software; COVID-19.

\begin{abstract}
Considering the public health emergency character of the pandemic; the urgency to adjust and rethink nursing care, with a view to overcoming or minimizing the clinical problems of people affected by Covid-19; and the importance of nurses' clinical decision-making in a quick, efficient and safe way, the objective was to develop an Android OS application capable of supporting the clinical reasoning of nurses, based on the steps of the nursing process for patients affected by COVID- 19. Methodological study, using the PMBOK for application development, following the steps organized into five groups of processes: 1. Initiation; 2. Planning; 3. Execution; 4. Monitoring; and 5. Closing. The development of the application project took place between the months of May and June 2020. The results and discussions show, at each stage, the feasibility of the application development, necessary resources and definition of the operating supporting system; the scope of the application was defined in order to enable the chain of ideas about nursing care; and the execution of activities necessary to achieve the project's objectives; performance monitoring strategies. It is concluded that the objective was fulfilled and that the authors' knowledge about Nursing information system programming and know-how was essential for the creation and projection of the Nursing COVID-19 application, in a fast time as required by the emergence of the pandemic and according to the needs of nurses.
\end{abstract}

Keywords: Nursing; Nursing process; Software design; COVID-19. 


\begin{abstract}
Resumen
Considerando el carácter de emergencia de salud pública de la pandemia; la urgencia de ajustes y repensar los cuidados de enfermería, con intento de disminuir los problemas clínicos de las personas con COVID-19; y la importancia de la toma de decisiones clínicas por parte del enfermero de manera rápida, eficiente y segura, el objetivo fue desarrollar una aplicación móvel de SO Android capaz de apoyar el razonamiento clínico del enfermero, basado en los pasos del proceso de enfermería para pacientes afectados por COVID-19. Estudio metodológico, utilizando el PMBOK para el desarrollo de la aplicación, siguiendo los pasos organizados en cinco grupos de procesos: 1. Iniciación; 2. Planificación; 3. Ejecución; 4. Seguimiento; y 5. Clausura. El desarrollo del proyecto de la aplicación se llevó a cabo entre los meses de mayo y junio de 2020. Los resultados y discusiones muestran, en cada etapa, la viabilidad del desarrollo de la aplicación, los recursos necesarios y la definición del sistema operativo de apoyo; se definió el alcance de la aplicación para posibilitar la cadena de ideas sobre el cuidado de enfermería; y la ejecución de las actividades necesarias para lograr los objetivos del proyecto; estrategias de seguimiento del desempeño. Se concluye que se cumplió el objetivo y que el conocimiento de los autores sobre programación y know-how de sistemas de información de Enfermería fue fundamental para la creación y proyección de la aplicación COVID-19 de Enfermería, en un tiempo rápido como lo requiere la pandemia y según las necesidades de las enfermeras.
\end{abstract}

Palabras clave: Enfermería; Proceso de enfermería; Diseño de software; COVID-19.

\title{
1. Introdução
}

Os vírus da família Coronavírus podem causar infecções respiratórias nos seres humanos, sendo o mais recémdescoberto deles o Novo Coronavírus (SARS-CoV-2) (World Health Organization [WHO], 2020).

A COVID-19 é transmitida principalmente por gotículas de secreção respiratória, contato físico e potencialmente pela via fecal-oral, causando os principais sinais e sintomas como febre, tosse, mialgia ou fadiga, além de diminuição do paladar. Há relatos de pacientes que não desenvolveram sintomas respiratórios, mas apresentaram disfunções múltiplas dos órgãos (Jin et al., 2020; Robba et al., 2020). A síndrome respiratória aguda grave (SARS-CoV), causada pela infecção do Novo Coronavírus em humanos, resulta em desfechos clínicos variados e em respostas humanas diversas, exigindo dos profissionais de saúde grande nível de atenção e agilidade no raciocínio clínico (Alhazzani et al., 2020).

A pandemia de COVID-19 (Coronavírus Disease 2019) impôs novos desafios aos profissionais e pressão adicional aos serviços de saúde de todo o mundo, com aumento da demanda por leitos em unidades de internação clínica e de terapia intensiva (Department of Health. New York State [NYSDOH], 2020). A equipe de enfermagem, por representar a maior parcela da força de trabalho em saúde, possui papel fundamental no tratamento e na reabilitação dos pacientes acometidos pela COVID-19, sendo este cuidado baseado em conhecimentos técnicos e em evidências científicas (Wang, Wang, Zeng, Wu \& Sun, 2019).

As enfermeiras, como líderes das equipes de enfermagem, possuem a responsabilidade de planejar a assistência, acompanhar sua execução e avaliar os resultados, utilizando o processo de enfermagem como principal ferramenta. Entende-se o processo de enfermagem como "uma etapa sistematizada, utilizada para orientar os cuidados dos profissionais de enfermagem e a documentação de sua prática profissional", descrito por cinco fases interrelacionadas: histórico de enfermagem, diagnósticos de enfermagem, planejamento de enfermagem, prescrição de enfermagem e avaliação de enfermagem (Conselho Federal de Enfermagem [COFEN], 2009).

O raciocínio clínico, base para o desenvolvimento do processo de enfermagem, depende de uma série de processos intelectuais complexos, que as enfermeiras usam na identificação de situações que demandam atendimento de enfermagem, e na seleção de ações necessárias para o alcance dos melhores resultados em saúde (Luria, Cervera \& Tsvetkov, 1981; Carvalho, Kamakura \& Morais, 2017). Neste sentido, o desenvolvimento de sistemas de suporte ao raciocínio clínico de enfermeiras deve ser uma preocupação de pesquisadores, podendo adotar as Tecnologias da Informação e Comunicação (TICs) como estratégias para potencializar todo processo.

As TICs possuem papel fundamental na estruturação e organização de dados em saúde, promovendo a disseminação do conhecimento científico e garantindo agilidade no acesso às informações (Carvalho et al., 2017). Baseadas em soluções 
tecnológicas e informatizadas, elas buscam agilizar processos em sistemas que envolvam pessoas (Biz, Azzolim \& Neves, 2016).

Essas TICs são efetivadas através da utilização de softwares, instruções escritas em linguagem de máquina que, quando executadas, fornecem funções e desempenhos desejados, podendo ser divididos em básico (sistemas operacionais), ferramentas (editores de textos, planilhas) ou aplicativos (baseados nas necessidades de usuários específicos) (Pressman, 2011; Silva, 2009).

Nos dias atuais, diversas TICs são projetadas para a utilização em smartphones, considerados computadores compactos, com grande capacidade de armazenamento de dados, que podem ser gerenciados pelos usuários através dos aplicativos, tendo a grande vantagem de permitirem o acesso às informações em qualquer hora ou lugar (Tibes, Dias, \& ZemMascarenhas, 2014).

Estas características atribuem aos smartphones grande potencial para suportarem aplicativos em saúde, capazes de facilitar o processo de consulta às informações pertinentes ao processo de tomada de decisão clínica.

Dado o caráter de emergência de saúde pública da pandemia; a urgência para se ajustar e repensar os cuidados de enfermagem, com vistas a se debelar ou minimizar os agravos clínicos das pessoas acometidas por COVID-19; e a importância da tomada de decisão clínica dos enfermeiros de forma rápida, eficiente e segura, este estudo objetivou desenvolver um aplicativo em SO Android capaz de fornecer suporte ao raciocínio clínico de enfermeiras, baseado nas etapas do processo de enfermagem para pacientes acometidos pela COVID-19.

\section{Metodologia}

Trata-se de um estudo metodológico, com foco no desenvolvimento de software (Polite \& Beck, 2018). Foram seguidas as etapas do Guia PMBOK, metodologia clássica utilizada no desenvolvimento de softwares e que busca a compreensão do problema a ser abordado, os requisitos e restrições e o processo de implementação e avaliação (Project Management Institute. [PMI], 2017).

As etapas do Guia PMBOK descrevem as boas práticas para o desenvolvimento de projetos, organizadas em cinco grupos de processos: 1. Iniciação; 2. Planejamento; 3. Execução; 4. Monitoramento; e 5. Encerramento. O desenvolvimento do projeto do aplicativo ocorreu entre os meses de maio e junho de 2020.

\section{Resultados e Discussão}

A organização do estudo de acordo com os grupos de processos permitiu a elaboração de um planejamento conciso e objetivo, além de nortear todo o processo de execução do projeto de software. Essas características foram fundamentais para impor agilidade e garantir a disponibilização do aplicativo em cerca de trinta dias após a ideia inicial. O desenvolvimento das atividades em cada grupo de processo será descrito a seguir.

\section{Iniciação}

Os processos de iniciação são utilizados a fim de definir um novo projeto a partir da autorização para o seu início. Nesta etapa, os pesquisadores envolvidos discutiram sobre a viabilidade do desenvolvimento do aplicativo, recursos necessários e definição do sistema operacional de suporte. Optou-se pelo desenvolvimento de um aplicativo Android devido à sua abrangência e, apesar de outros SOs serem utilizados em smartphones no Brasil, o tempo necessário para realizar a programação inviabilizaria o projeto. Cabe ressaltar que, no ano de 2020, cerca de $90 \%$ dos smartphones no Brasil utilizavam o sistema operacional (SO) Android, sendo o principal meio de se atingir número expressivo de usuários para os aplicativos 
(Moura \& Camargo, 2020). Os custos para o desenvolvimento, o registro e a divulgação do aplicativo foram estimados e, por não haver tempo suficiente para solicitação de apoio financeiro, foram assumidos integralmente pelos pesquisadores. Carece atenção por parte das instituições públicas de fomento para a necessidade de mecanismos para disponibilização ágil de recursos para pesquisas, além de incentivo da participação do setor privado nesse processo (Tenorio, 2017). Por outro lado, pesquisadores e lideranças da enfermagem necessitam buscar espaços de articulação política que favoreçam a apresentação das demandas específicas desta categoria (Marziale \& Mendes, 2006).

\section{Planejamento}

Os processos de planejamento são necessários para a definição do escopo do projeto e refinar seus objetivos. O escopo do aplicativo foi definido de maneira a possibilitar o encadeamento de idéias sobre a assistência de enfermagem aos pacientes acometidos pela COVID-19, conforme apresentado na Figura 1.

A tela de entrada do aplicativo, além de fornecer acesso às demais funções, foi planejada para apresentar informações sobre os desenvolvedores e um formulário para envio de dúvidas sobre o funcionamento ou sugestões pelos usuários. Estas funcionalidades permitiram a criação de um canal de comunicação entre os desenvolvedores e os usuários, de maneira a melhorar a utilização do aplicativo e contribuir para o seu aprimoramento.

Para o menu principal foram planejados acessos às informações sobre o Novo Coronavírus, a fisiopatologia da COVID-19 e as referências utilizadas no desenvolvimento do projeto. Estes conteúdos teóricos são fundamentais para apoiar o processo de decisão clínica no processo de enfermagem (Luiz et al, 2020).

Figura 1: Diagrama do escopo do aplicativo

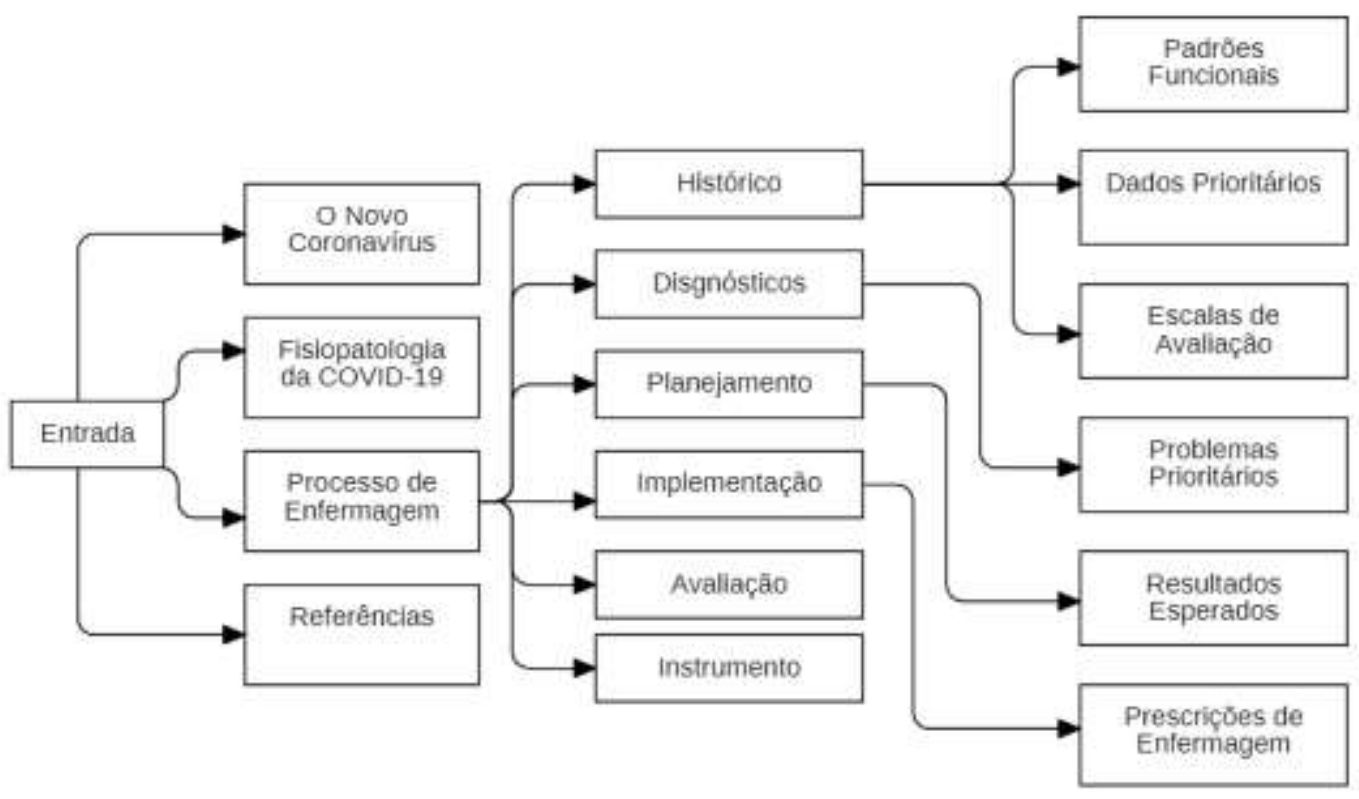

Fonte: Construído pelos autores.

$\mathrm{O}$ acesso às etapas do processo de enfermagem compõe a parte mais complexa do planejamento, pois, para cada etapa, foram definidos conjuntos de informações sobre a assistência de enfermagem focada no perfil clínico dos pacientes acometidos pela COVID-19.

Planejou-se a elaboração de vídeos com definições teóricas de cada uma das etapas do processo de enfermagem, contextualizando as especificidades dos pacientes acometidos pela COVID-19. Estes vídeos foram omitidos do fluxograma do 
escopo por questões de clareza. Levando-se em conta as diferentes realidades dos serviços de saúde brasileiros e a carência de sistemas de registro do processo de enfermagem em muitas instituições (Patrício, Machiavelli, Maia, \& Navaes, 2011), foi planejado o desenvolvimento de um instrumento de registro para ser disponibilizado, gratuitamente, através do aplicativo.

\section{Execução}

Os processos de execução são aqueles utilizados para executar atividades necessárias ao alcance dos objetivos do projeto. As seguintes etapas foram definidas durante a execução:

- Revisão da literatura: busca de evidências sobre surgimento e disseminação do Novo Coronavírus, sobre a fisiopatologia da COVID-19 e sobre a assistência de enfermagem aos pacientes acometidos pela doença. A partir dos artigos científicos levantados nas bases internacionais, foi possível definir conjuntos de dados prioritários para serem investigados no histórico de enfermagem, os principais diagnósticos e resultados esperados, além de prescrições de enfermagem mais prevalentes. Nessa busca foram identificados 51 diagnósticos de enfermagem, 36 resultados esperados, 125 indicadores clínicos e 76 intervenções de enfermagem. Estas informações apontaram, também, para a necessidade de incorporação de três escalas importantes durante a avaliação dos pacientes: a escala de Braden para avaliação dos riscos de desenvolvimento de lesões por pressão, a escala de Agitação-Sedação de Richmond (RASS) e a escala de Avaliação da Disfunção Orgânica Sequencial (SOFA). A utilização deste conjunto de informações fornece suporte ao raciocínio clínico dos enfermeiros tanto no tratamento quanto na prevenção de disfunções e contribui para a otimização do fluxo dos pacientes nos serviços de saúde (Barros et al, 2020). De maneira a contemplar a utilização de um referencial teórico para nortear o processo de coleta de dados de enfermagem, foi incluída no projeto a apresentação dos Padrões Funcionais de Saúde, identificados nos estudos de Marjory Gordon (Gordon, 1994).

- Programação: o aplicativo foi desenvolvido por um dos pesquisadores, sob a plataforma de programação Android Studio, escrito na linguagem Java, utilizando as melhores práticas preconizadas pela empresa Google, desenvolvedora do SO Android. O layout das telas foi planejado de maneira a permitir fácil leitura dos conteúdos, sendo escolhidas como base as cores azul (\#648EB) e branco (\#FFFFFF), de maneira a diminuir impactos sobre a visão dos usuários, já que o uso prolongado dos dispositivos móveis contribui para o aumento da fadiga visual (Park, 2017). Para as etapas do processo de enfermagem foram desenvolvidas apresentações em vídeo, com imagem e som, para correlacionar cada etapa às particularidades da COVID-19. Os vídeos foram incorporados ao código-fonte do aplicativo, de maneira a eliminar a necessidade de acesso à internet durante sua execução. Uma seleção de telas do aplicativo é apresentada na Figura 2. Um instrumento para registro das etapas do processo de enfermagem foi desenvolvido no software de código aberto, LibreOffice, compondo uma planilha com listas suspensas, que facilitam o seu preenchimento, sendo disponibilizado, gratuitamente, através do aplicativo, assim como um vídeo-tutorial sobre sua utilização (Figura 3). A utilização de um instrumento baseado em software para registro do processo de enfermagem contribuiu para a agilidade, diminuindo o tempo demandado aos enfermeiros para esta função e disponibilizando a estes profissionais maior tempo para o cuidado direto aos pacientes (Tannure, 2015). 
Figura 2: seleção de telas do aplicativo Enfermagem COVID-19.

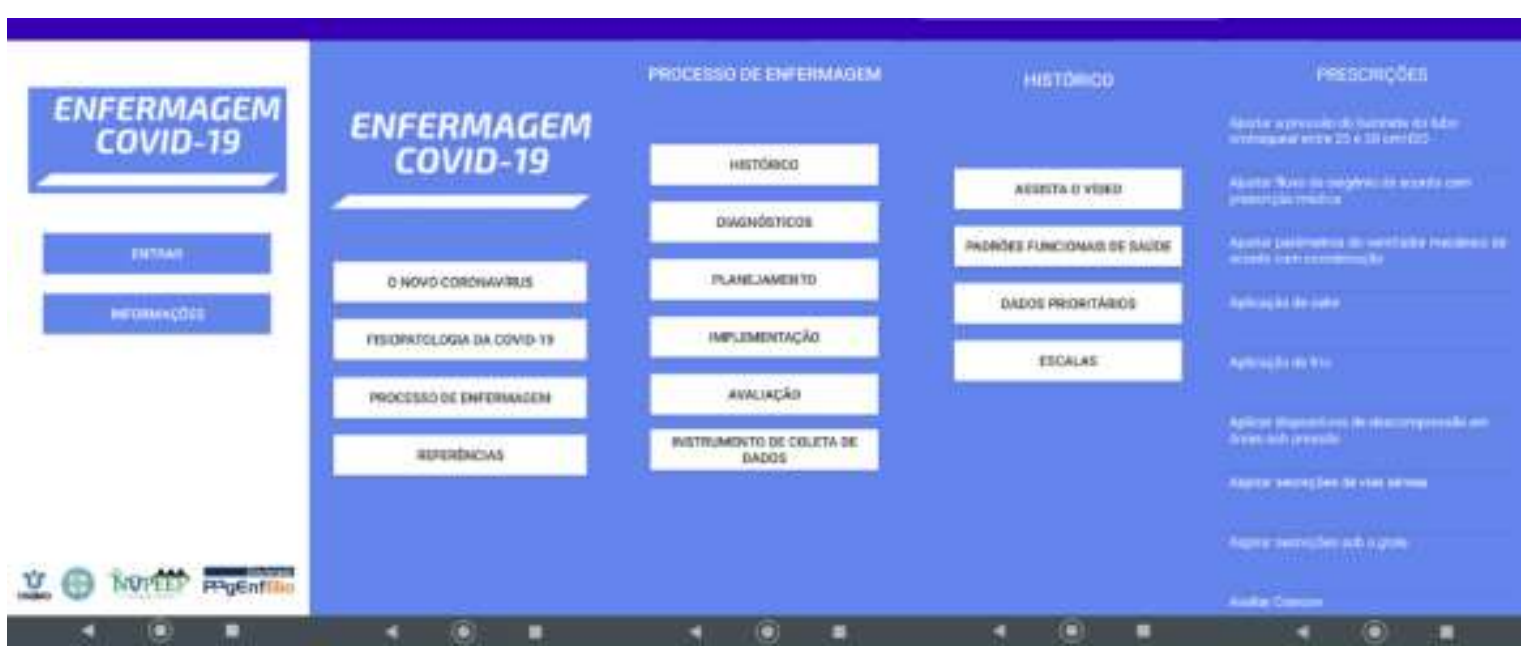

Fonte: Construído pelos autores.

Figura 3: Instrumento de registro das etapas do processo de enfermagem.

\begin{tabular}{|c|c|c|c|c|c|c|c|c|}
\hline \multirow[t]{2}{*}{8} & c & D & $E$ & $F$ & G & H & \multirow{2}{*}{\multicolumn{2}{|c|}{ ACCÖES DE ENFERMAGEM }} \\
\hline & PROBLEMA DE ENFERMAGEM / RESULTADO & \multicolumn{5}{|c|}{ METAS } & & \\
\hline Prob & Constipaçâo & & & & & & Realizar massagem abdominal & \\
\hline Res & Funçăo Gastrointestinal & \multicolumn{5}{|c|}{ Meta } & Solicitar parecer de nutricionista & \\
\hline \multirow[t]{2}{*}{ Ind } & Frequencia des fezes & Aumentar & De & Para & Em & & \multicolumn{2}{|l|}{ Planejar a dieta } \\
\hline & & & 1 & 2 & 12 & $\mathrm{~h}$ & 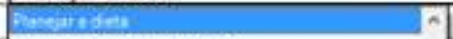 & \\
\hline \multirow[t]{2}{*}{ Ind } & Consistencia des Fezes & Aumentar & De & Para & Em & & \multirow{9}{*}{ 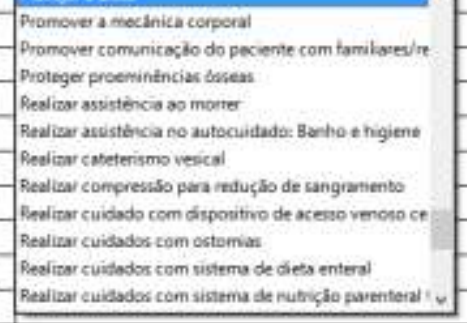 } & \\
\hline & & & 2 & 3 & 12 & $\mathrm{~h}$ & & \\
\hline Prob & Integridade Tissular Prejudicada & & & & & & & \\
\hline Res & Cicatrização de Feridas: Sequnda Intençāo & \multicolumn{5}{|c|}{ Meta } & & \\
\hline \multirow[t]{2}{*}{ Ind } & Quantidade de exsudato & Aumentar & De & Para & Em & & & \\
\hline & & & 3 & 4 & 12 & h & & \\
\hline \multirow[t]{2}{*}{ Ind } & Granulac:ä:0 & Aumentar & De & Para & Em & & & \\
\hline & & & 1 & 2 & 5 & dia & & \\
\hline Prob & Choque séptico & & & & & & & \\
\hline Res & Gravidade do Choque Septico & \multicolumn{5}{|c|}{ Meta } & & \\
\hline \multirow[t]{2}{*}{ Ind } & $\mathrm{pH}$ sanguineo Elevado & Aumentar & De & Para & Em & & & \\
\hline & & & 4 & 5 & 12 & $\mathrm{~h}$ & & \\
\hline \multirow[t]{2}{*}{ Ind } & Enchimento Capilar & Aumentar & De & Para & Em & & & \\
\hline & & & 2 & 3 & 12 & h & & \\
\hline Prob & Trocas gasosas comprometidas & & & & & & & \\
\hline Res & Perfusäo Tissular: Pulmonar & \multicolumn{5}{|c|}{ Meta } & & \\
\hline
\end{tabular}

Fonte: Construído pelos autores.

- Testes e revisões: testes foram realizados durante toda a etapa de programação, utilizando-se dois smartphones de modelos diferentes e um tablet. Estes procedimentos foram importantes para verificar o comportamento do aplicativo em diferentes ambientes e garantir a característica de adaptabilidade, não tendo sido identificada nenhuma dificuldade. Os conteúdos foram revisados independentemente pelos pesquisadores, a fim de serem identificados e corrigidos erros ortográficos e equívocos conceituais.

- Registro: o código-fonte do aplicativo, suas telas e conteúdos foram submetidos à avaliação para garantia do registro de autoria de software no Instituto Nacional da Propriedade Industrial (INPI), encontrando-se ainda em análise no momento de elaboração deste relato. 
- Divulgação: o aplicativo foi planejado e desenvolvido com a finalidade de ser disponibilizado, gratuitamente, na loja de aplicativos para smartphones da empresa Google (Google Play). Entretanto, após o cadastro na loja e submissão do código fonte, a empresa emitiu notificação informando a restrição de publicação de quaisquer aplicativos envolvendo a COVID-19 por pessoas ou entidades sem ligação com secretarias ou ministérios da saúde dos países. Tal restrição foi contornada com a aquisição de um domínio de internet e desenvolvimento de um website para disponibilização do aplicativo aos profissionais de enfermagem e estudantes interessados (Figura 4). A liberação do download do aplicativo, através do website, é realizada após um breve cadastro do usuário, a fim de permitir a contabilização dos acessos. A divulgação do website do aplicativo foi realizada em redes sociais e grupos de enfermagem, em aplicativos de mensagem e na Biblioteca Virtual e Saúde (Conceição \& Tonini, 2020).

Figura 4: Website para divulgação e download do aplicativo Enfermagem COVID-19.
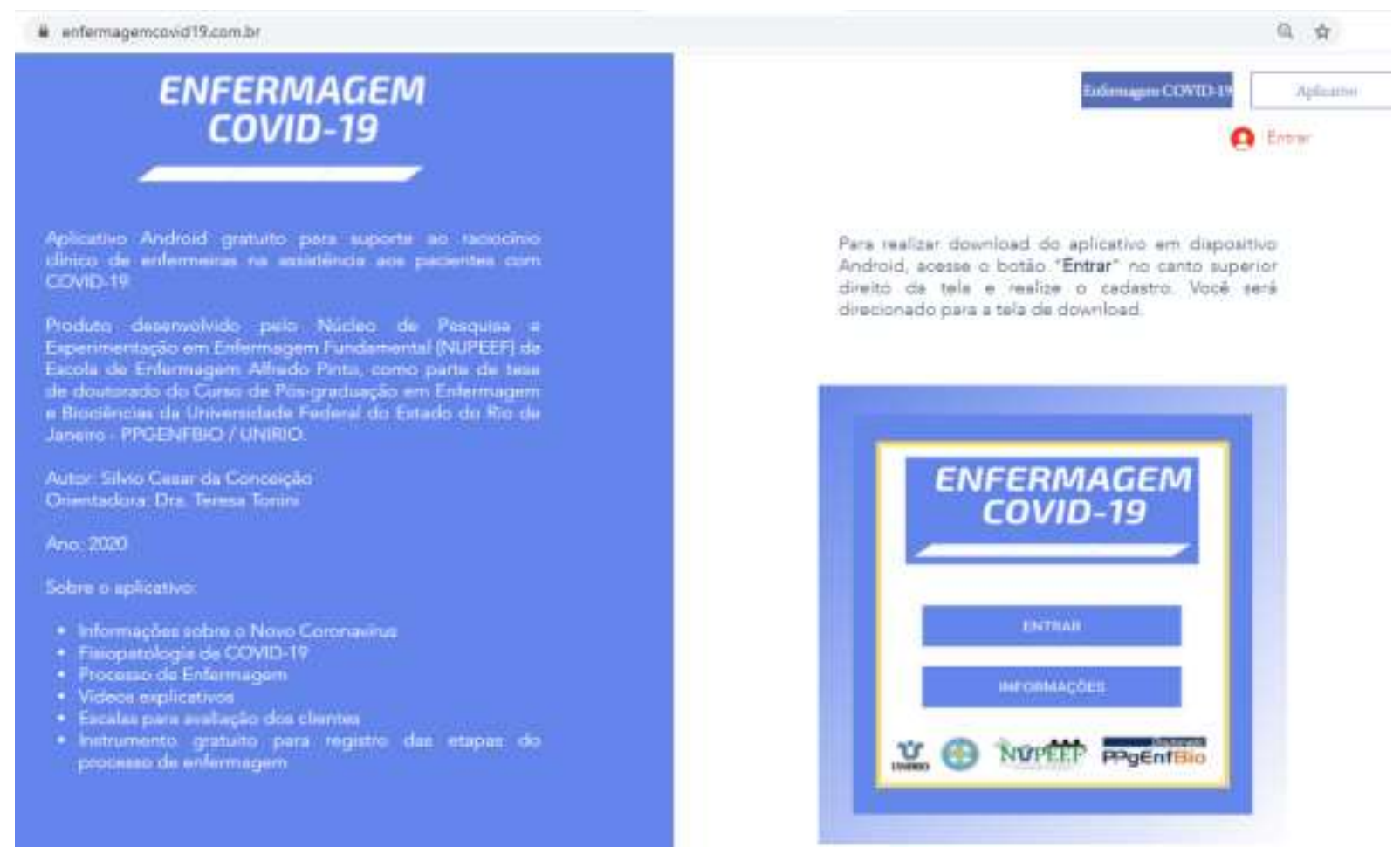

Fonte: Construído pelos autores.

\section{Monitoramento}

Os processos de monitoramento são utilizados para acompanhar, analisar e controlar o progresso e o desempenho do projeto. Para esta etapa, foram utilizadas como estratégias o número de acessos no website, o número de downloads do aplicativo, além de mensagens com dúvidas e sugestões enviadas através do formulário disponibilizado na tela de informações do aplicativo. Entre os dias 30 de junho e 31 de dezembro de 2020 foram realizadas 3587 visitas ao website, 957 downloads do aplicativo e 36 envios do formulário de dúvidas e sugestões. Entre as principais demandas por parte dos usuários, destacam-se os pedidos para desenvolvimento de aplicativo compatível com outro sistema operacional, inclusão de maior número de diagnósticos de enfermagem e dúvidas em relação ao funcionamento do instrumento de registro disponibilizado.

\section{Encerramento}

Os processos de encerramento finalizam as atividades do projeto, por decisão dos autores ou por perda da utilidade do produto. No desenvolvimento do aplicativo Enfermagem COVID-19, a etapa de encerramento ainda não pode ser concluída 
devido ao fato de continuar sendo necessária a interação constante com os usuários finais. Ademais, ainda não foi atingido nenhum dos critérios considerados para se definir a obsolescência de um software: existência de falhas, finalização do suporte ou perda da utilidade (Sandborn et al, 2007; Nielsen, 2012).

\section{Conclusão}

$\mathrm{Na}$ área de saúde, o desenvolvimento de aplicativos tem gerado melhor armazenamento de informações, maior redução de tempo e menor demanda de deslocamentos dos profissionais. Pode-se afirmar que o conhecimento dos autores sobre programação de sistema de informação e saber-fazer de Enfermagem foi fundamental para a criação e projeção do aplicativo Enfermagem COVID-19, em tempo veloz como requer a emergência de uma pandemia e de acordo com as necessidades e demandas do público a que se destina.

Embora se tenha alcançado o objetivo de apoiar as enfermeiras no raciocínio clínico para o processo de enfermagem elaborado aos pacientes acometidos pela COVID-19, é importante que estudos de validação sejam realizados a posteriori, em especial o de usabilidade. Isso poderá gerar a melhoria do design do escopo inicial, das funções e desempenho dessa nova tecnologia.

Ademais, se configurou como contribuição ao campo dos saberes e das práticas na área de Enfermagem, uma vez que proporciona aprimorar a coordenação dos cuidados e a aplicação de evidências científicas, por meio de o processo de enfermagem.

Recomenda-se a realização de outros estudos na área de desenvolvimento de software para apoio ao raciocínio clínico, focando em diferentes especialidades da enfermagem.

\section{Referências}

WHO. World Health Organization. (2020). Q\&A on coronaviruses (COVID-19). https://www.who.int/emergencies/diseases/novel-coronavirus-2019/questionand-answers-hub/q-a-detail/q-a-coronaviruses

Departamente of Health. New York State. (2020). COVID-19 Frequently Asked Questions. https://coronavirus.health.ny.gov/system /files/documents/2020/04/doh_covid19_faqs_updated_041720_2.pdf

Alhazzani, W., Møller, M. H, Arabi, Y. M, Loeb, M., Gong, M. N, Fan, E., Oczkowski, S., Levy, M. M, Derde, L., Dzierba, A., Du, B., Aboodi, M., Wunsch, H., Cecconi, M., Koh, Y., Chertow, D. S, Maitland, K., Alshamsi, F., Belley-Cote, E., Greco, M., \& Rhodes, A. (2020). Campanha Sobrevivendo à Sepse: diretrizes sobre o manejo de adultos gravemente enfermos com Doença do Coronavírus 2019 (COVID-19). Medicina de terapia intensiva, 46 (5), 854-887. https://doi.org/10.1007/s00134-020-06022-5.

Jin, Y., Yang, H., Ji, W., Wu, W., Chen, S., Zhang, W., \& Duan G. (2020). Virology, Epidemiology, Pathogenesis, and Control of COVID-19. Viruses. 12, 372. https://pubmed.ncbi.nlm.nih.gov/32230900/

Robba, C., Battaglini, D., Ball, L., Patroniti, N., Loconte, M., Brunetti, I., Pelosi, P. (2020). Distinct phenotypes require distinct respiratory management strategies in severe COVID-19. Respiratory Physiology \& Neurobiology. 279. https://www.ncbi.nlm.nih.gov/pmc/articles/PMC7211757/

Wang, H., Wang, H., Zeng, T., Wu, X., \& Sun, H. (2019). Holistic care for patients with severe coronavirus disease: An expert consensus, International. Journal of Nursing Sciences. https://doi.org/10.1016/j.ijnss.2020.03.010.

COFEN. Conselho Federal de Enfermagem. Resolução 358/2009. http://www.cofen.gov.br/resoluo-COFEN-3582009_4384.html.

Luria, A. R., Cervera, N. M., \& Tsvetkova, L. S. (1981). La Resolución de problemas y sus trastornos. Fontanella.

Carvalho, E. C., Kamakura, A. R. S. O., \& Morais, S. C. R. V. (2017). Raciocínio clínico em enfermagem: estratégias de ensino e instrumento de avaliação. Revista Brasileira de Enfermagem, 70(3). https://www.scielo.br/pdf/reben/v70n3/pt_0034-7167-reben-70-03-0662.pdf

Barra, D. C. C., Paim, S. M. S., Sasso, G. T. M. D., \& Colla, G. W. (2018). Métodos para desenvolvimento de aplicativos móveis em saúde: Revisão integrativa de literatura. Texto \& Contexto - Enfermagem, .26(4). https://dx.doi.org/10.1590/0104-07072017002260017 
Biz, A. A., Azzolim, R., Neves A. J. W. A. (2016). Estudo dos Aplicativos para Dispositivos Móveis com Foco em Atrativos Turísticos da Cidade de Curitiba (PR). Anais do Seminário da ANPTUR. https://www.anptur.org.br/anais/anais/files/13/468.pdf

Pressman, R. S. (2011). Engenharia de software: uma abordagem profissional. (7a ed.), Bookman.

Silva, R. F. L, (2009). e-RH em um ambiente global e multicultural. Senac-DF.

Tibes, C. M. S., Dias, J. D., \& Zem-Mascarenhas, S. H. (2014). Aplicativos móveis desenvolvidos para a área da saúde no Brasil: revisão integrativa da literatura. Rev Min Enferm. abr/jun; 18(2): 471-478. http://www.dx.doi.org/10.5935/1415-2762.20140035

Polite, D., Beck C. (2018) Fundamentos de Pesquisa em Enfermagem: Avaliação de Evidências para a Prática da Enfermagem. (9a ed.) Artmed.

Project Management Institute. (2017). A Guide to the Project Management Body of Knowledge (PMBOK® Guide) - Seventh Edition.

Moura, L., \& Camargo, G. (2020). Impacto econômico e social do Android no Brasil. https://www.bain.com/pt-br/insights/economic-and-social-impact-ofandroid-in-brazil/

Tenório, M., Mello, G. A., \& Viana, A. L. D. (2017). Políticas de fomento à ciência, tecnologia e inovação em saúde no Brasil e o lugar da pesquisa clínica. Ciênc. saúde colet. 22 (5). https://doi.org/10.1590/1413-81232017225.33342016.

Marziale, M. H. P., \& Mendes, I. A. C. (2006). O investimento em pesquisas na área da saúde: termos de referência para o desenvolvimento científico e tecnológico brasileiro. Rev. Latino-Am. Enfermagem vol.14 no.2 Ribeirão Preto Mar./Apr. http://dx.doi.org/10.1590/S0104-11692006000200001.

Luiz, F. S., Vasconcellos, L. J., Barbosa, A. C. S., Paiva, A. C. P. C., Santos, K. B., Sanhudo, N. F., \& Carbogim, F. C. (2020) Papel do pensamento crítico na tomada de decisão pelo enfermeiro: revisão integrativa. REAS/EJCH. 38. e1763. https://doi.org/10.25248/reas.e1763.2020.

Patrício, C. M., Machiavelli, J. L., Maia, M. M., \& Navaes, M. A. (2011). O prontuário eletrônico do paciente no sistema de saúde brasileiro: uma realidade para os médicos? Scientia Medica; 21(3), 121-131. https://revistaseletronicas.pucrs.br/ojs/index.php/scientiamedica/article/viewFile/8723/6722.

Barros et al. Contribuições da rede de pesquisa em processo de enfermagem para assistência na pandemia de COVID-19. Rev. Bras. Enferm. 73(2). https://doi.org/10.1590/0034-7167-2020-0798.

Gordon, M. (1994). Nursing diagnosis: Process and application. Mosby.

Park., Y. H., An., C. M., Moon, S. J. (2017) Effects of visual fatigue caused by smartphones on balance function in healthy adults. J Phys Ther Sci. Feb; 29(2): 221-223. https://dx.doi.org/10.1589\%2Fjpts.29.221.

Tannure, M. C., Lima, A. P. S., Oliveira, C. R., Lima, S. V., Chianca, T. C. M. (2015) Processo de Enfermagem: comparação do registro manual versus eletrônico. J. Health Inform. 7(3): 69-74. http://www.jhi-sbis.saude.ws/ojs-jhi/index.php/jhi-sbis/article/viewFile/337/377.

Conceição, S. C., Tonini, T. (2020) Enfermagem COVID-19: aplicativo android gratuito [Programa de computador]. Núcleo de Pesquisa e Experimentação em Enfermagem Fundamental (NUPEEF) da Escola de Enfermagem Alfredo Pinto. UNIRIO. https://pesquisa.bvsalud.org/portal/resource/pt/lis-47730.

Sandborn, P. et al. (2007) A Taxonomy and Evaluation Criteria for DMSMS Tools. Databases and Services. http://escml.umd.edu/Papers/AgingAircraft07Taxonomy.pdf.

Nielsen, J. (2012) Usability 101: introduction to usability. https://www.nngroup.com/articles/usability-101-introduction-to-usability/. 